\title{
MAXIMUM MODULUS CONVEXITY AND THE LOCATION OF ZEROS OF AN ENTIRE FUNCTION
}

\author{
FARUK F. ABI-KHUZAM
}

(Communicated by Irwin Kra)

\begin{abstract}
Let $f$ be an entire function with non-negative Maclaurin coefficients and let $b(r)=r\left(r f^{\prime}(r) / f(r)\right)^{\prime}$. It is shown that if all the zeros of $f$ lie in the angle $|\arg z| \leq \delta$, where $0<\delta \leq \pi$, then $\limsup _{r \rightarrow \infty} b(r) \geq \frac{1}{4} \operatorname{cosec}^{2} \frac{1}{2} \delta$. In particular, we always have $\lim \sup _{r \rightarrow \infty} b(r)>\frac{1}{4}$ for such functions.
\end{abstract}

\section{INTRODUCTION}

Let $f$ be an entire function and let $M(r)=M(r, f)=\sup _{|z|=r}|f(z)|$ be its maximum modulus. It is known that the function

$$
b(r)=d^{2} \log M(r) / d(\log r)^{2}
$$

exists and is continuous except at isolated points and $b(r) \geq 0$ by Hadamard's convexity theorem. Some time ago Hayman [2] showed that in certain situations a little more can be said about $b(r)$. Specifically Hayman showed that if $f$ is transcendental, then $\lim \sup _{r \rightarrow \infty} b(r) \geq A_{0}$ where $A_{0}>.18$. Hayman conjectured that $\frac{1}{4}$ is the best possible value of $A_{0}$ but this was disproved by Kjellberg [3]. At about the same time Boichuck and Gol'dberg [1] proved that the best possible value of $A_{0}$ is indeed $\frac{1}{4}$ if discussion is restricted to entire functions with positive coefficients. They also showed that more information about $b(r)$ may be obtained if the class of functions under consideration is further restricted. In fact they proved that if $f(z)=\sum_{k=0}^{\infty} a_{k} z^{n_{k}}$ is entire and $A=\lim \sup _{k \rightarrow \infty}\left(n_{k+1}-n_{k}\right)$ then $A_{0} \geq \frac{1}{4} A^{2}$ when $a_{k}>0$ for all $k$. Thus the presence of gaps in the Maclaurin series of $f$ tends to increase the size or growth of $b(r)$.

In this note we consider the connection between the size of $b(r)$ and the location of the zeros of $f$. It turns out that there is a simple and direct relationship between the size of $b(r)$ as measured by $\lim \sup b(r)$ and the location of the zeros relative to the negative $x$-axis. The smallest value of $\lim \sup b(r)$ occurs when all but a finite number of the zeros lie on or in the direction of the

Received by the editors July 8, 1988 and, in revised form, December 1, 1988. $30 \mathrm{D} 35$.

1980 Mathematics Subject Classification (1985 Revision). Primary 30D20, 30D15; Secondary 
negative $x$-axis, and it increases as we swing the zeros away from the negative $x$-axis. Our main result may be stated as follows.

Theorem 1. Let $f$ be an entire function with non-negative Maclaurin coefficients and suppose that the equation $f(z)=w$ has infinitely many roots in the angle $|\arg z| \leq \delta$, where $0<\delta \leq \pi$. Then

$$
\limsup _{r \rightarrow \infty} b(r) \geq \frac{1}{4} \operatorname{cosec}^{2} \frac{1}{2} \delta
$$

In particular if $f$ is any transcendental entire function with non-negative coefficients then

$$
\limsup _{r \rightarrow \infty} b(r) \geq \frac{1}{4} \text {. }
$$

Note that (1.3) is Hayman's $\frac{1}{4}$-conjecture for functions with positive coefficients.

A result slightly more general than Theorem 1 may also be obtained by our method.

Theorem 2. Let $f$ be an entire function with non-negative Maclaurin coefficients and $g$ an entire function satisfying $M(r, g)=o(f(r))$ as $r \rightarrow \infty$. Assume that

$$
\frac{1}{4 \alpha}=\limsup _{r \rightarrow \infty} b(r)<+\infty \text {. }
$$

Then for every $\delta \in\left(0,2 \sin ^{-1} \sqrt{\alpha}\right)$ the equation $f(z)=g(z)$ has at most a finite number of roots in $|\arg z| \leq \delta$.

In the original paper [2], Hayman showed that when $f$ is a polynomial then limsup $\operatorname{sum}_{r \rightarrow \infty} b(r)=0$; but $\sup _{r>0} b(r) \geq A_{0}>.18$ if $f$ is not of the form $c z^{m}$. Hayman asked whether there exist entire functions other than $c z^{m}$ which satisfy $b(r+0)=b(r-0)=0$ for a value of $r>0$. An example of such a function was constructed by London [4]. Our next result shows that when the coefficients are non-negative, the vanishing of $b(r)$ at one point $r>0$ implies $f(z)=c z^{m}$.

Theorem 3. Let $f$ be an entire function with non-negative Maclaurin coefficients. Then

(a) $\limsup _{r \rightarrow \infty} b(r)=0$ if and only if $f$ is a polynomial;

(b) there exists $R>0$ such that $b(R)=0$ if and only if $f(z)=c z^{m}$;

(c) if $f$ is a polynomial other than $c z^{m}$ then $\sup _{r>0} b(r) \geq \frac{1}{4}$

and equality holds if and only if $f(z)=c z^{n}(1+z / a)$ where $c>0, a>0$ and $n$ is a non-negative integer.

\section{AN AUXILIARY LEMMA}

An entire function $f$ with non-negative coefficients cannot vanish on the positive $x$-axis unless $a_{n}=0$ for all $n$ where $a_{n}$ is its $n$th Maclaurin coefficient. Thus if one of its coefficients is positive, then $f(r)>0$ for all $r>0$ and, 
by continuity, we may find an open set containing the positive $x$-axis where $f$ never vanishes. Is there a simple way of describing some such set? The following Lemma which is fundamental to our proofs arose as an answer to the above question.

Lemma 1. Let $f(z)=\sum_{n=0}^{\infty} a_{n} z^{n}$ be an entire function satisfying $a_{n} \geq 0$ for $n=0,1,2, \cdots$ and let $b(r)$ be defined by (1.1). If $r \geq 0$ and $z=r e^{i \theta}$ then we have

$$
f^{2}(r)-|f(z)|^{2} \leq\left(4 \sin ^{2} \frac{1}{2} \theta\right) f^{2}(r) b(r) .
$$

Proof. We have

$$
\begin{aligned}
f^{2}(r)-|f(z)|^{2} & =\sum_{n=0}^{\infty} r^{n} \sum_{k=0}^{n} a_{n-k} a_{k}\left(1-e^{i(n-2 k) \theta}\right) \\
& =\sum_{n=0}^{\infty} r^{n} \sum_{k=0}^{n} a_{n-k} a_{k} \cdot 2 \sin ^{2}(n-2 k) \frac{1}{2} \theta \\
& \leq\left(2 \sin ^{2} \frac{1}{2} \theta\right) \sum_{n=0}^{\infty} r^{n} \sum_{k=0}^{n} a_{n-k} a_{k}(n-2 k)^{2} \\
& =\left(4 \sin ^{2} \frac{1}{2} \theta\right) f^{2}(r) b(r) .
\end{aligned}
$$

The second equality above is due to the fact that the sum is real; while the inequality in the third step is a result of $|\sin (m t)| \leq|m||\sin t|$ which is valid for all integers $m$ and all real $t$. The last equality is obtained as follows: Since $a_{n} \geq$ 0 we have $M(r)=f(r)$ for all $r \geq 0$ and so $b(r)$ can be expressed explicitly in terms of $f(r)$ and its first two derivatives. Indeed using the definition (1.1) of $b(r)$ we have

$$
f^{2}(r) b(r)=r^{2} f(r) f^{\prime \prime}(r)+r f(r) f^{\prime}(r)-\left(r f^{\prime}(r)\right)^{2} .
$$

If the terms appearing on the righthand side of (2.2) are expressed as series we obtain

$$
\begin{aligned}
f^{2}(r) b(r) & =\sum_{n=0}^{\infty} r^{n} \sum_{k=0}^{n} a_{n-k} a_{k}\{k(k-1)+k-k(n-k)\} \\
& =\sum_{n=0}^{\infty} \frac{1}{2} r^{n} \sum_{k=0}^{n} a_{n-k} a_{k}\left\{k^{2}-k(n-k)+(n-k)^{2}-(n-k) k\right\} \\
& =\frac{1}{2} \sum_{n=0}^{\infty} a_{n} \sum_{k=0}^{n} a_{n-k} a_{k}(n-2 k)^{2}, \quad(r \geq 0) .
\end{aligned}
$$

This finishes the proof of Lemma 1.

We now turn to the proofs of our results. We shall prove Theorem 3 first since we are going to use one of its assertions in the proof of Theorem 1.

Proof of Theorem 3. (a). Let $f(z)=\sum_{n=0}^{\infty} a_{n} z^{n}$ be an entire function with non-negative coefficients and suppose that $\lim _{\sup _{r \rightarrow \infty}} b(r)=0$ where $b(r)$ is 
defined in (1.1). Let $\epsilon$ be a positive number less than 1 . Then there exists $R>0$ such that $b(r)<\frac{1}{4}(1-\epsilon)$ for all $r>R$. If this, together with $\sin ^{2} \frac{1}{2} \theta \leq$ 1 , is used in (2.1) we obtain

$$
|f(z)| \geq \sqrt{\epsilon} f(r) \quad(|z|=r>R) .
$$

Now if $a_{n}=0$ for all $n \geq 1$, there is nothing to prove. Otherwise $a_{n}>0$ for at least one value of $n \geq 1$ in which case $f(r) \rightarrow \infty$ as $r \rightarrow \infty$ and then, (2.4) implies that $|f(z)| \rightarrow \infty$ as $|z| \rightarrow \infty$. But then $f$ must be a polynomial being an entire function with a pole at $\infty$. Conversely, if $f$ is a polynomial then by [2, p. 212] $\lim \sup _{r \rightarrow \infty} b(r)=0$ and this does not require the hypothesis of positivity of coefficients.

(b). Suppose that $b(R)=0$ for some $R>0$. Then (2.1) gives $\left|f\left(R e^{i \theta}\right)\right| \geq f(R)$ for all $\theta$; and since the opposite inequality is always true under our assumptions (non-negativity of coefficients) we must have

$$
|f(z)|=f(R) \quad \text { for all }|z|=R .
$$

We may assume that $f(R) \neq 0$ since otherwise $f(z) \equiv 0$. Let $\left\{z_{1}, z_{2}, \ldots, z_{p}\right\}$ be the possible zeros of $f$ in $0<|z|<R$, necessarily finite in number since otherwise $f(z) \equiv 0$. Let $m \geq 0$ be the multiplicity of the possible zero of $f$ at the origin. Put $\phi_{\alpha}(z)=R(z-\alpha) /\left(R^{2}-\bar{\alpha} z\right)$ and note that $\left|\phi_{\alpha}(z)\right|=1$ when $|z|=R$. Now put $g(z)=f(z) / z^{m} \prod_{k=1}^{p} \phi_{z_{k}}(z)$. Then $g$ is analytic and never vanishes in $|z| \leq R$, and $|g(z)|=\left|z^{-m} f(z)\right|=R^{-m} f(R)$ on $|z|=R$. By the maximum principle applied to $g$ and to $1 / g$, we conclude that $g$ must be a constant in $|z| \leq R$. That is $g(z)=c$ where $|c|=R^{-m} f(R)$. It follows that

$$
f(z)=c z^{m} \prod_{k=1}^{p} \phi_{z_{k}}(z) \quad \text { for all }|z| \leq R,
$$

and so for all $z \neq z_{k}$. But $f$ has no poles and hence we must have $f(z)=c z^{m}$. Since the converse is trivial the proof of part (b) is complete.

(c). Suppose that $f(z)$ is a polynomial other than $c z^{m}$ with positive coefficients. Then $f$ has zeros $\left\{z_{1}, \bar{z}_{1}, \ldots, z_{N}, \bar{z}_{N}\right\}$ away from the origin. If these zeros are ordered so that $0<\theta_{1} \leq \theta_{2} \leq \cdots \leq \theta_{N} \leq \pi$ where $\theta_{k}=\arg z_{k}$ then by using $z_{1}$ in (2.1) we obtain $1 \leq\left(4 \sin ^{2} \frac{1}{2} \theta_{1}\right) b\left(r_{1}\right) \leq 4 b\left(r_{1}\right)$ where $r_{1}=\left|z_{1}\right|$. Hence $\sup _{r>0} b(r) \geq \frac{1}{4} \csc ^{2} \frac{1}{2} \theta_{1} \geq \frac{1}{4}$.

For the remaining parts of (c) note first that if $f(z)=c z^{n}(1+z / a)$ with positive $c$ and $a$ then $b(r)=r a /(r+a)^{2}$ from which it follows immediately that $\sup _{r>0} b(r)=\frac{1}{4}$. Suppose next that $f$ is an entire function with positive coefficients and that $\sup _{r>0} b(r)=\frac{1}{4}$. If $z=r e^{i \theta}$ is a zero of $f$ away from the origin then $(2.1)$ gives $1 \leq\left(4 \sin ^{2} \frac{1}{2} \theta\right) b(r) \leq \sin ^{2} \frac{1}{2} \theta$ and this implies that $\theta=$ $\pi$. Thus all the zeros of $f$ must lie on the negative $x$-axis. Since $\log M(r, f)=$ $O(\log r)^{2}$ we may write $f(z)=c z^{n} \prod_{k=1}^{N}\left(1+z / z_{k}\right)$ where $z_{k}>0$ and $1 \leq$ $N \leq \infty$. But then we will have $b(r)=\sum_{k=1}^{N}\left(r z_{k} /\left(r+z_{k}\right)^{2}\right)$ and $b\left(z_{1}\right)$ will be 
greater than $\frac{1}{4}$ unless $N=1$. Hence $N=1$ and $f$ has the desired form. This finishes the proof of (c) and the proof of Theorem 3 is complete.

Proof of Theorem 1. Let $\delta \in(0, \pi]$ and assume that $f$ has an infinite number of zeros in the angle $|\arg z| \leq \delta$. If we denote these zeros by $z_{n}=r_{n} e^{i \theta_{n}}$ where $r_{n}$ increases to $\infty$, then $\left|\theta_{n}\right| \leq \delta$ and $\sin ^{2} \frac{1}{2} \theta_{n} \leq \sin ^{2} \frac{1}{2} \delta$. Using $z=z_{n}$ in (2.1) we obtain $1 \leq\left(4 \sin ^{2} \frac{1}{2} \delta\right) b(r)$ and this implies (1.2). To prove (1.3) we may assume that $\limsup _{r \rightarrow \infty} b(r)<+\infty$. Then $\log M(r, f)=O(\log r)^{2}$ and since $f$ is now assumed to be transcendental it must have an infinity of zeros. Of course these zeros lie in the angle $|\arg z| \leq \pi$ and so taking $\delta=\pi$ we have $\csc ^{2} \frac{1}{2} \delta=1$ and (1.3) follows from (1.2).

Proof of Theorem 2. Let $f$ be an entire function with non-negative Maclaurin coefficients and suppose that (1.4) holds true. Then $\alpha \in(0,1]$ by $(1.3)$, and $\log M(r, f)=O(\log r)^{2}$. By part (a) of Theorem 3, (1.4) also implies that $f$ is not a polynomial and so $f$ is transcendental. Let $g$ be an entire function satisfying $M(r, g)=o(f(r))$ and assume that the equation $f(z)=g(z)$ has an infinite number of roots in $|\arg z| \leq \delta$, where $\delta \in\left(0,2 \sin ^{-1} \sqrt{\alpha}\right)$. Denote these roots by $w_{n}=r_{n} e^{i \theta_{n}}$ so that $r_{n}=\left|w_{n}\right|$ increases to infinity and $\left|\theta_{n}\right| \leq \delta$. Then $\sin ^{2} \frac{1}{2} \theta_{n} \leq \sin ^{2} \frac{1}{2} \delta<\alpha$ and, by Lemma 1 ,

$$
f^{2}\left(r_{n}\right)-\left|g\left(w_{n}\right)\right|^{2} \leq\left(4 \sin ^{2} \frac{1}{2} \theta_{n}\right) f^{2}\left(r_{n}\right) b\left(r_{n}\right) \leq\left(4 \sin ^{2} \frac{1}{2} \delta\right) f^{2}\left(r_{n}\right) b\left(r_{n}\right) .
$$

Since $f(r) \rightarrow \infty$ as $r \rightarrow \infty$ we have $f\left(r_{n}\right) \neq 0$ for all large $n$. Of course $\left|g\left(w_{n}\right)\right|=o\left(f\left(r_{n}\right)\right)$ as $n \rightarrow \infty$. Dividing by $f\left(r_{n}\right)$ and passing to the limit as $n \rightarrow \infty$ we obtain $\left(4 \sin ^{2} \frac{1}{2} \delta\right) \lim \sup _{r \rightarrow \infty} b(r) \geq 1$. This last inequality implies that $\lim \sup _{r \rightarrow \infty} b(r)>1 / 4 \alpha$ a contradiction to (1.4). Hence at most a finite number of the roots of the equation $f(z)=g(z)$ lie in the angle $|\arg z| \leq \delta$.

\section{EXAMPLES}

We define

$$
g(z)=\prod_{k=1}^{\infty}\left(1+z e^{-k^{2}}\right) .
$$

Then $g$ has all its zeros on the negative $x$-axis, its Maclaurin coefficients are non-negative and by [2, p. 213] lim $\sup _{r \rightarrow \infty} b(r, g)=\frac{1}{4}$. Let $n$ be a positive integer and put $f(z)=f(z ; b)=g\left(z^{n}\right)$. It is easy to verify that $b(r, f)=$ $n^{2} b\left(r^{n}, g\right)$ and so $\limsup _{r \rightarrow \infty} b(r, f)=n^{2} / 4$. Thus for the function $f, \alpha=$ $1 / n^{2}$ and the zeros nearest to the $x$-axis lie on the ray $\pi / n$. We have to compare $\pi / n$ with $2 \sin ^{-1} \sqrt{\alpha}$. The inequality $2 / n<2 \sin ^{-1}(1 / n) \leq \pi / n$ is easily verified for $n \geq 1$. Equality on the right side holds only for $n=1$. This shows that the constant $2 \sin ^{-1} \sqrt{\alpha}$ in Theorem 2 is best possible when $\alpha=1$. We conjecture that it is best possible for all $\alpha \in(0,1)$. 


\section{REFERENCES}

1. V. S. Boichuk and A. A. Gol'dberg, The three-lines theorem (Russian), Mat. Zametki, 15 (1974), 45-53.

2. W. K. Hayman, Note on Hadamard's convexity theorem, Proc. of Pure Math., Vol. 11, Entire functions and related parts of analysis, Amer. Math. Soc., 210-213, 1968.

3. B. Kjellberg, The convexity theorem of Hadamard-Hayman, Proc. of the Sympos. in Math., Royal Institute of Technology, Stockholm (June 1973), 87-114.

4. R. R. London, A note on Hadamard's three circles theorem, Bull. London Math. Soc., 9 (1977), 182-185.

Department of Mathematical Sciences, King Fahd University of Petroleum and Minerals, Dhahran 31261, Saudi ARabia 